complete set of states in elliptic coordinates: C. M. Dutta, N. C. Dutta, and T. P. Das, to be published.

${ }^{6}$ H. P. Kelly, Phys. Rev. Lett. 23, 445 (1969), and Phys. Rev. A 1,274 (1970).

${ }^{7} \mathrm{C}$. W. Townes and A. L. Schawlow, Microwave Spectroscopy (McGraw-Hill, New York, 1955).

${ }^{8}$ N. F. Ramsey, Molecular Beams (Oxford Univ., Oxford, England, 1956)。

${ }^{9}$ P. E. Cade and W. M. Huo, J. Chem. Phys. 47, 614 (1967).

${ }^{10}$ C. E. Bender and E. R. Davidson, Phys. Rev。 $\underline{183}$, 23 (1969).

${ }^{11}$ G. Herzberg, Spectra of Diatomic Molecules (Van Nostrand, New York, 1950).

${ }^{12}$ C. Matsubara, N. C. Dutta, T. Ishihara, and T. P. Das, Phys. Rev. A 1, 561 (1970)。

${ }^{13}$ The total energy of $\mathrm{HF}$ is given by $E=-\mathrm{I}_{0} \mathrm{P} .+E\left(\mathrm{~F}^{+7}\right)$ $+E(\mathrm{H})-D_{e}$, where I.P. denotes the sum of the ionization potentials, $24.2051 \pm 0.005$ a.u., of the neutral fluorine atom up to the innermost two electrons [taken from B. Edlén, in Handbuch der Physik, edited by S. Flügge (Springer, Berlin, Germany, 1964), Vol. 27, p. 198], and $E\left(\mathrm{~F}^{+7}\right)$ denotes the total nonrelativistic energy, -75.5317 a.u., of the He-like ion Itaken from C. L. Pekeris, Phys. Rev。112, 1649 (1958)]. Since we are interested only in the nonrelativistic energy, the first number has to be corrected for relativistic effects which amount to 0.0133 a.u. [H. Hartmann and E. Clementi, Phys. Rev. 133, A1295 (1964)] arising almost entirely from the first two electrons beyond the Helike shell. $E(\mathrm{H})$ denotes the nonrelativistic energy of the hydrogen atom which is -0.5 a.u., and $D_{e}$ denotes the dissociation energy of $\mathrm{HF}$ which is equal to 0.225 \pm 0.0004 a.u. [Ref. 11, and P. G. Wilkinson, Astrophys. J. 138, 778 (1963)].

${ }^{14}$ The correlation energy of the neon atom has been calculated recently utilizing the same basis set; T. Lee, N. C. Dutta, and T. P. Das, to be published.

\title{
LINE SHAPES IN SOFT X-RAY ABSORPTION SPECTRA OF SOLIDIFIED RARE GASES
}

\author{
R. Haensel and G. Keitel \\ II. Institut für Experimentalphysik der Universität Hamburg, Hamburg, Germany
}

and

C. Kunz and P. Schreiber

Deutsches Elektronen-Synchrotron, Hamburg, Germany

(Received 11 June 1970)

\begin{abstract}
We have performed measurements of the shape of the absorption lines due to transitions from the $2 s$ shell in solid Ne and the $3 s$ shell in solid Ar. It was found that the asymmetric and the "window" line shapes seen in the absorption spectra of the gases are preserved in the solids.
\end{abstract}

In the soft $x$-ray region the absorption spectra are mainly due to electronic transitions from inner (core) shells. Those transitions to dis crete final states, which normally show up in the absorption spectra as lines of enhanced absorption, may however interfere with the underlying continuum transitions from the outer shells to final states far above threshold.

This configuration interaction between the discrete and continuum states is responsible for special asymmetric and "window" line shapes near the resonance frequency. In the rare gases those line profiles have been extensively investigated at the U. S. National Bureau of Standards ${ }^{1}$ by means of synchrotron radiation.

Theoretically these line shapes are described by an expression for the absorption cross section of Fano, 2

$$
\sigma=\sigma_{a} \frac{(q+\epsilon)^{2}}{1+\epsilon^{2}}+\sigma_{b}
$$

$\sigma_{a}$ is that part of the total continuum absorption which interferes with the discrete excitation, $\rho^{2}=\sigma_{a} /\left(\sigma_{a}+\sigma_{b}\right)$ is the fraction of the interfering continuum in the total absorption continuum, and $q$ is a profile index. The photon energy $\epsilon$ $=2\left(E-E_{0}\right) / \Gamma$ is measured in units of the broadening parameter $\Gamma / 2$ with its zero at a nominal resonance energy $E_{0}$. The continuum absorption cross sections $\sigma_{a}$ and $\sigma_{b}$ are assumed to be constant over the energy region where the continuumdiscrete interaction takes place.

Besides lifetime broadening due to the configuration interaction which is included in Fano's parametrization formula, the effect of another broadening mechanism such as phonons has been introduced into Eq. (1) by folding with a Gaussian profile of suitable width. ${ }^{3}$

Experimental evidence for asymmetric profiles in solids has been reviewed by $\mathrm{Phillips}^{4}$ and Greenaway and Harbeke ${ }^{5}$ and recently reported by Brown et al. ${ }^{6}$ However the reported spectra do not allow a comparison of the spectra of one material in both the atomic and the solid state. 
Therefore we felt it would be interesting to investigate the corresponding transitions in the rare gases in both states of aggregation. We have measured previously the absorption lines associated with $4 d$ transitions of solid $\mathrm{Xe}, 3 d$ transitions of solid $\mathrm{Kr},{ }^{7}$ and $2 p$ transitions of solid Ar. ${ }^{8}$ As in the gas, lines of the enhanced absorption type have been found, which were attributed to exciton excitations in the solid. This paper presents an investigation of the $2 s$ transitions in solid $\mathrm{Ne}$, for which Codling, Madden, and Ederer ${ }^{9}$ have found asymmetric lines in the corresponding gas spectra, and the $3 s$ transitions in solid Ar, for which Samson, ${ }^{10}$ Madden and Codling, ${ }^{11}$ and Madden, Ederer, and Codling $^{12}$ have detected window lines in the gas spectra.

The 7.5-GeV electron synchrotron DESY was used as light source ${ }^{13}$ for a 1-m Rowland monochromator. The 2400-line/mm grating was illuminated at a grazing angle of about $220 \mathrm{mrad}$. An open photomultiplier (Bendix M 306) served as the detector. Ne was evaporated onto a $\sim 2000-$ $\AA$-thick aluminum foil and Ar onto a $\sim 1000-\AA-$ thick antimony foil. The substrates were cooled in a He cryostat.

In the case of $\mathrm{Ne}$ the temperature was kept at $6^{\circ} \mathrm{K}$ and in the case of Ar it was kept at about $15^{\circ} \mathrm{K}$. The substrates also served as filters to reduce higher-order light. In addition to the spectra of the solids we have measured the line series of the $2 s$ transitions in Ne gas. The energy position of these lines as given by Codling, Madden, and Ederer ${ }^{9}$ served as calibration marks. The absorption coefficients were determined in arbitrary units with a relative accuracy of $3 \%$.

Figure 1 shows the absorption spectrum of solid and gaseous $\mathrm{Ne}$ in the energy range 44 to $50 \mathrm{eV}$. The spectral resolution of the monochromator, as derived from the width of the zeroorder reflection, is $0.04 \mathrm{eV}$ for gaseous $\mathrm{Ne}$ and $0.025 \mathrm{eV}$ for solid Ne. Therefore, the structure of the solid-Ne spectrum is not expected to be broadened by our monochromator. As in the case of gaseous $\mathrm{Ne}$, one recognizes asymmetric lines with similar line shape in solid Ne. The onset of the lines in solid $\mathrm{Ne}$ is shifted to higher energies, the lines are broadened and only two show up. Whereas the gas lines are sitting on a flat continuum background, the lines in solid $\mathrm{Ne}$ are superimposed onto a broad hump in the continuum absorption background, probably due to high-lying valence-band transitions.

Figure 2 shows the absorption spectrum of solid Ar in the energy range 26 to $29 \mathrm{eV}$. The spectral resolution is better than $0.015 \mathrm{eV}$. For comparison, the absorption section of the gas spectrum as measured by Madden, Ederer, and Codling ${ }^{12}$ is included. The absorption spectrum of solid Ar shows two "window" lines which are less pronounced than those in the gas. The width compared with the gas lines has not in-

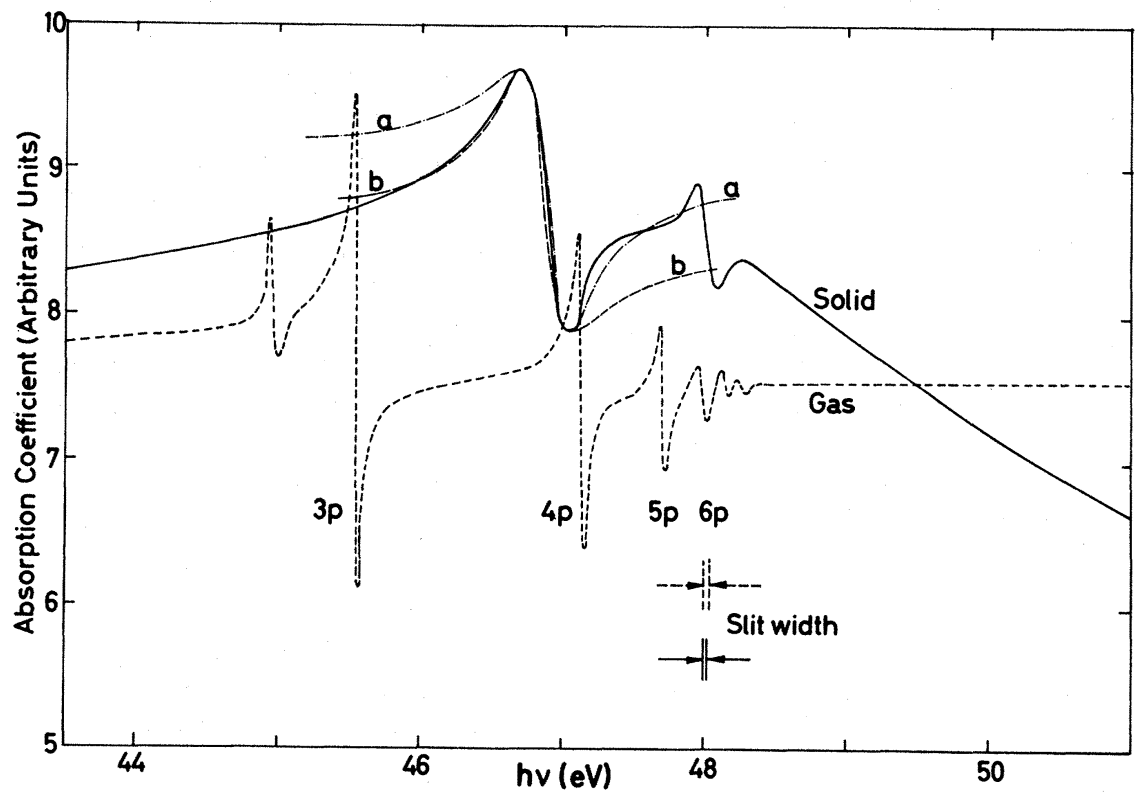

FIG. 1. Absorption spectrum of solid (solid curve) and gaseous (dashed curve) Ne. Theoretical curves $a$ and $b$ are included, according to Eq. (1) with two different sets of parameters (see Table I). 


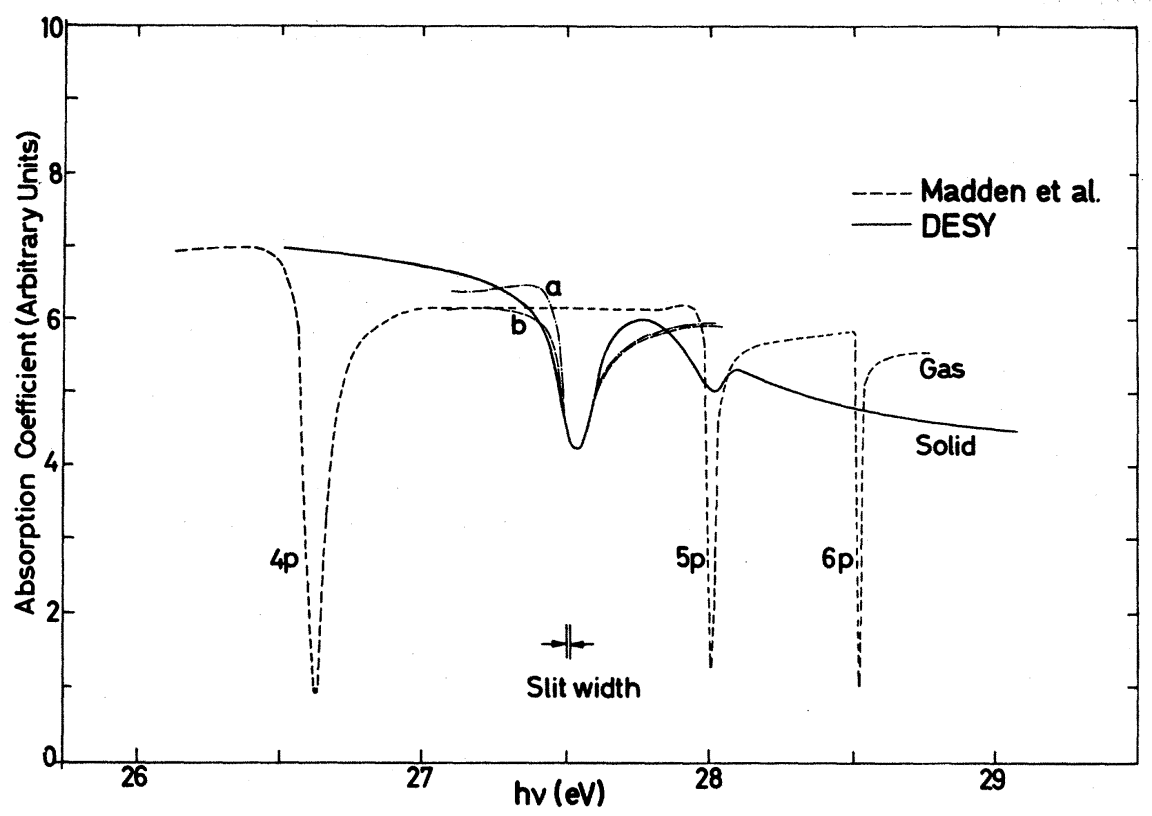

FIG. 2. Absorption spectrum of solid Ar (solid curve). The absorption spectrum of gaseous Ar as given by Madden, Ederer, and Codling (Ref. 12) is included (dashed curve). Also theoretical curves $a$ and $b$ are included, according to Eq. (1) with two different sets of parameters (see Table I).

creased as much as in the case of Ne. Again, there is a shift to higher energies and the relative distance of the lines has decreased appreciably. As in Ne, the gas lines are superimposed onto a flat background whereas the lines in solid Ar are sitting on a hump which is obviously due to valence-band transitions. It has also been seen in reflectance measurements of solid $\mathrm{Ar}$ at the high-energy end of valence-band transitions. ${ }^{8}$

In Table I the line-shape parameters for the first lines of the $\mathrm{Ne}$ and Ar gas spectra are given after Madden, Ederer, and Codling. ${ }^{12}$ By simply folding the gas spectra with Gaussians of various widths $^{3}$ we have tried to match the profiles of

Table I. Line-shape parameters according to Eq. (1) for Ne gas [after Codling, Madden, and Ederer (Ref. 9)] and different fits to Ne solid (see Fig. 1), for Ar gas [after Madden, Ederer, and Codling (Ref. 12)] and different fits to Ar solid (see Fig. 2).

\begin{tabular}{llllll}
\hline \hline & & $\begin{array}{c}E_{0} \\
(\mathrm{eV})\end{array}$ & $\begin{array}{c}\Gamma \\
(\mathrm{eV})\end{array}$ & $\rho^{2}$ & $q$ \\
\hline Ne gas & & 45.55 & 0.013 & 0.7 & -1.6 \\
Ne solid & Fit $a$ & 46.90 & 0.34 & 0.125 & -0.76 \\
& Fit $b$ & 46.81 & 0.36 & 0.077 & -1.31 \\
Ar gas & & 26.614 & 0.08 & 0.86 & -0.22 \\
Ar solid & Fit $a$ & 27.515 & 0.12 & 0.32 & -0.39 \\
& Fit $b$ & 27.525 & 0.13 & 0.30 & -0.20 \\
\hline
\end{tabular}

the first absorption lines of the solids. This procedure did not lead to a satisfactory result. We have also tried to fit the line shapes with Eq. (1). It turns out, however, in solid $\mathrm{Ne}$, that the parameters necessary to fit the line shape at the low-energy side (fit $b$ ) are different from those necessary for an optimal fit $(a)$ on the high-energy side. This discrepancy seems due to the fact that the background continuum absorption is not constant as it is in the gas. For solid Ar, fit $a$ and fit $b$ give equally good descriptions of the profiles.

Although a quantitative description of the line profiles with Fano's parametrization formula does not lead to a satisfying result, a comparison of the line-shape parameters for the gas and the solid lines shows that in going from gas to solid the fraction $\rho^{2}$ of the interfering background decreases, the width $\Gamma$ of the lines increases, and the line-shape parameter $q$ has the same sign and the same order of magnitude.

We have considered whether the large linewidths, especially in $\mathrm{Ne}$, could be due to impurities or imperfections in the samples. After having reproduced these spectra several times with samples prepared under different vacuum conditions and with different evaporation speeds we do not believe that this is the case.

\footnotetext{
${ }^{1}$ R. P. Madden and K. Codling, in Autoionization;
} 
Astrophysical, Theoretical and Laboratory Experimental Aspects, edited by A. Temkin (Mono Book Corp., Baltimore, Md., 1966), p. 129.

${ }^{2}$ U. Fano, Phys. Rev。124, 1966 (1961).

${ }^{3}$ K. P. Jain, Phys. Rev. 139, A544 (1965).

${ }^{4} \mathrm{~J}$. C. Phillips, in Solid State Physics, edited by H. Ehrenreich, F. Seitz, and D. Turnbull (Academic, New York, 1966), Vol. 18.

${ }^{5}$ D. L. Greenaway and G. Harbeke, Optical Properties and Band Structures of Semi-conductors (Pergamon, New York, 1968).

${ }^{6}$ F. C. Brown, Ch. Gähwiller, H. Fujita, N. Carrera, and W. Scheifley, in Proceedings of the Tenth European Congress on Molecular Spectroscopy, Liège, Belgium,
1969 (unpublished).

${ }^{7}$ R. Haensel, G. Keitel, P. Schreiber, and C. Kunz, Phys. Rev. 188, 'nme Mnnn

${ }^{8}$ R. Haensel, G L E T T E R S och, M. Skibowski, and P. Schreiber, Phys. Rev. Lett. 23, 1160 (1969).

${ }^{9}$ K. Codling, R. P. Madden, and D. L. Ederer, Phys. Rev. 155, 26 (1967).

${ }^{10}$ J. A. R. Samson, Phys. Rev. 132, 2122 (1963).

${ }^{11}$ R. P. Madden and K. Codling, Phys. Rev. Lett. 10 , 516 (1963).

${ }^{12}$ R. P. Madden, D. L. Ederer, and K. Codling, Phys. Rev. 177, 136 (1969)。

${ }^{13}$ R. Haensel and C. Kunz, Z. Angew. Phys. 37, 276 (1966).

\title{
CHARGE STATES AND EXCITATION OF FAST HEAVY IONS PASSING THROUGH SOLIDS: A NEW MODEL FOR THE DENSITY EFFECT*
}

\author{
Hans D. Betz and Lee Grodzins \\ Massachusetts Institute of Technology, Cambridge, Massachusetts 02139 \\ (Received 13 April 1970)
}

\begin{abstract}
The theory of Bohr and Lindhard for electron capture and loss by heavy ions penetrating through solids has been modified. It is concluded that the difference in the average equilibrium charge of fast ions traversing solids and dilute gases is mainly due to Auger processes which occur after the ions leave the solid. The charge states inside solids are -in disagreement with the deductions of Bohr and Lindhard-not much larger than those in gases.
\end{abstract}

The mean ionization $\bar{q}$ of energetic heavy ions which penetrate through matter reaches an equilibrium value determined by the competition between capture and loss of electrons. It has been known for many years that $\bar{q}$ is greater when the ions traverse solids rather than gases. Lassen ${ }^{1}$ found this effect in a study of fission fragments, and many further experiments ${ }^{2-6}$ revealed even more pronounced differences. An example is given in Fig. 1, which shows the equilibrium charge spectrum of $12-\mathrm{MeV}$ iodine ions following passage through oxygen gas and a carbon foil. ${ }^{6}$ The resulting mean charges are 6 and 12 , respectively. ${ }^{7,8}$. It has always been assumed that such investigations measure essentially the equilibrium charge distribution inside the stripper.

The difference $\Delta \bar{q}$ between the mean charge in a solid compared with a gas stripper was explained by Bohr and Lindhard ${ }^{9}$ (BL) in a pioneering paper of 1954, from which we quote below and to which we refer hereafter as the BL theory. In this generally accepted model, those collisions of the ions with the target atoms which do not produce ionization result in excitation of the ion's most loosely bound electron. In a dilute gas stripper, de-excitation back to the ion's ground state takes place before the next collision occurs. In a solid, however, the collision rate is too high for such radiative de-excitation to be important. Rather the collisions constantly increase the excitation of the outermost electron until that electron is lost. This reasoning applies consecutively to inner electrons and, consequently, the charge of the ion will steadily increase until the capture cross section becomes large enough to match the probability for loss of the excited electron. A new equilibrium value of the mean ionization is finally maintained by direct competition between capture and loss. Mainly one or, at most, a very few electrons will be in states of fairly high excitation and "an electron captured into an excited state will be lost from the same state." Thus, "suppression of readjustment of ion excitation" is expected to be the principal mechanism for increasing the charge inside solids to the values observed after the ions passed through the target, though Bohr and Lindhard also state that "the high excitation of the ions in solids may result in a subsequent emission of electrons from the ions immediately after their escape into vacuum, which increases the mean charge to a certain extent."

Several recent investigations have shown that 